\title{
A novel dual oxidase maturation factor 2 gene mutation for congenital hypothyroidism
}

\author{
RU-HAI YI ${ }^{1}$, WEN-BIN ZHU ${ }^{2}$, LI-YONG YANG ${ }^{1}$, LAN LAN $^{3}$, YAO CHEN $^{2}$, \\ JIN-FU ZHOU ${ }^{2}$, JING WANG ${ }^{2}$ and YUE-QING SU ${ }^{2}$ \\ ${ }^{1}$ Department of Endocrinology and Metabolism, The First Affiliated Hospital, Fujian Medical University, \\ Fuzhou, Fujian 350001; ${ }^{2}$ Center of Neonatal Screening, and ${ }^{3}$ Bloodletting Room, Fujian Maternity \\ and Child Health Care Hospital, Fuzhou, Fujian 350001, P.R. China
}

Received September 18, 2012; Accepted November 23, 2012

DOI: $10.3892 /$ ijmm.2012.1223

\begin{abstract}
Congenital hypothyroidism $(\mathrm{CH})$ is caused by thyroid hormone deficiency present at birth. DUOXA2 (dual oxidase maturation factor 2 ) is one of the prerequisites for thyroid hormone synthesis. The present study explored the novel mutations of DUOXA2 in $\mathrm{CH}$ patients. Genomic DNA was extracted from peripheral blood of 47 unrelated $\mathrm{CH}$ patients, their family members and 100 healthy controls. All 6 exons and their flanking sequences of the DUOXA2 gene were PCR amplified and sequenced. Sequencing results were compared with the standard. Compound heterozygosity with DUOXA2 gene mutations at c.413-414ins A (Y138X) and c.738C $>\mathrm{G}$ (Y246X) was identified in one patient, and absent in 100 healthy controls. Among them, the c.413-414insA (Y138X) mutation was a novel one. The patient with the c.413-414insA (Y138X) mutation had mild CH symptoms. This study is the first to report a novel c.413-414insA (Y138X) mutation for $\mathrm{CH}$, thereby expanding the mutational spectrum of the DUOXA2 gene.
\end{abstract}

\section{Introduction}

Congenital hypothyroidism $(\mathrm{CH})$, also known as cretinism, is a disease caused by thyroid hormone deficiency present at birth (1). $\mathrm{CH}$ can lead to delayed growth and development and mental retardation; $85 \%$ of $\mathrm{CH}$ cases are secondary to thyroid developmental abnormalities such as athyreosis, hypoplastic or ectopic gland, and the remaining $15 \%$ of $\mathrm{CH}$ cases are due to genetic defects of thyroid hormone synthesis within a structurally normal gland (1). Although the molecular mechanisms of thyroid gland development and thyroid hormone synthesis and function have been studied

Correspondence to: Professor Yue-Qing Su, Center of Neonatal Screening, Fujian Maternity and Child Health Care Hospital, 18 Daoshan Road, Fuzhou, Fujian 350001, P.R. China

E-mail:syq0506@hotmail.com

Key words: congenital hypothyroidism, dual oxidase maturation factor 2, mutation extensively, the genetic pathogenesis remains relatively unclear (2).

A key step in thyroid hormone biosynthesis is iodine organification, which can only be successfully accomplished in the presence of $\mathrm{H}_{2} \mathrm{O}_{2}$. In thyroid follicular cells, Duox2 proteins are glycosylated and bound in the follicular cavity at the apical membrane, catalyzing the formation of $\mathrm{H}_{2} \mathrm{O}_{2}$, which is involved in the key step in thyroid hormone synthesis - iodine organification (3). Therefore, the normal functioning of Duox 2 is one of the prerequisites for thyroid hormone synthesis. Several studies have suggested that mutations in the Duox2 gene can lead to $\mathrm{CH}$ (4-9).

DUOXA2, a DUOX maturation factor, plays an important role in Duox 2 maturation and activation, thus determining the normal functionality of Duox2 (10). Genetic defects in DUOXA2 lead to defects in DUOX2 protein expression, which in turn lead to $\mathrm{CH}$ (11). Thus, DUOXA2 is an important entry point for molecular genetic studies of $\mathrm{CH}$. This study explored the novel mutations of DUOXA2 in $\mathrm{CH}$ patients

\section{Materials and methods}

Ethics statement. Approval was obtained from the local Ethics Committee. All participants provided written informed consent prior to enrollment in the study.

Patients. All $47 \mathrm{CH}$ cases were first screened at the Fujian Neonatal Disease Screening Center between 1998 and 2011. The patients, 27 males and 20 females, were diagnosed and followed up by the Neonatal Department, Maternal and Child Health Hospital of Fujian Province. Thyroid agenesis, hypoplasia, or ectopic thyroid abnormalities were excluded in all objects through ECT or ultrasound. As controls, 100 unrelated healthy controls were enrolled in this study in the First Affiliated Hospital of Fujian Medical University.

Genomic DNA extraction. Venous blood ( $2 \mathrm{ml}$ ) was obtained from the $47 \mathrm{CH}$ patients, their parents, siblings (if any), and unrelated healthy controls. Sodium citrate was added as anticoagulant. Genomic DNA (30-80 ng/ $\mu \mathrm{l}$ ) was extracted according to the manufacturer's instruction (Tiangen Biotech Co.) and stored at $-20^{\circ} \mathrm{C}$. 
Table I. PCR primers and annealing temperatures for 6 exons of the DUOXA2 gene.

\begin{tabular}{|c|c|c|c|}
\hline Exon & Primer (5'-3') & Product length (bp) & Annealing temp $\left({ }^{\circ} \mathrm{C}\right)$ \\
\hline 1 & $\begin{array}{l}\text { F: CAGCCTTGTACGCAAAGAGA } \\
\text { R: CCССАСТCTACCTGCACTA }\end{array}$ & 289 & 57 \\
\hline 2 & $\begin{array}{l}\text { F: GTCTTGGGGACTCTGGTTT } \\
\text { R: ACCCCAGTTCCCTATTGTCC }\end{array}$ & 201 & 56 \\
\hline 3 & $\begin{array}{l}\text { F: CAGTGTCCCACCTCCCATA } \\
\text { R: CTCACCTAACCGGGGATCT }\end{array}$ & 272 & 57 \\
\hline 4 & $\begin{array}{l}\text { F: TTCCTGTCTGAATCCGCTTA } \\
\text { R: CATCCTCCCGCTCATACG }\end{array}$ & 356 & 58 \\
\hline 5 & $\begin{array}{l}\text { F: GGGGTAGGGATAAAGAAGAGC } \\
\text { R: AATCCTGTCTCCACCCTTAGC }\end{array}$ & 351 & 56 \\
\hline 6 & $\begin{array}{l}\text { F: GTTTGAGGCCAGAGTTCGAG } \\
\text { R: GGGGAAGGAGTCCAGATTG }\end{array}$ & 295 & 56 \\
\hline
\end{tabular}

F, forward; R, reverse.

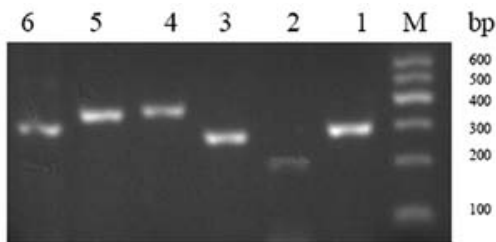

Figure 1. Agarose gel electrophoresis of target fragments of 6 exons in the DUOXA2 gene amplified by PCR. M, marker; lanes 1-6 are the PCR amplification products of 1-6 exons.

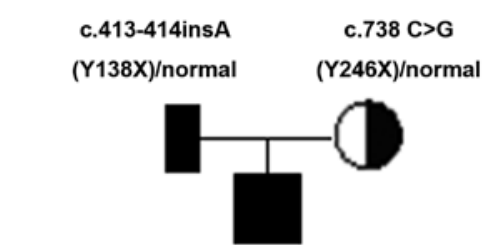

Patient c.413-414insA (Y138X)/c.738 C>G (Y246X)

Figure 2. Genotypes of the patient and the parents.
Primer design and PCR. All primers were designed to the flanking intron regions of the exons (1). The primers were synthesized by Shanghai Sangon Biotechnology Co., Ltd. The primer sequences and the length of the PCR products are shown in Table I. The PCR reaction volume was $50 \mu 1$, containing $2 \mu \mathrm{l}$ DNA template; $2 \mu \mathrm{l}$ upstream and downstream primers $(10 \mu \mathrm{mol} / \mu \mathrm{l})$, respectively; $4 \mu \mathrm{l}$ of $\mathrm{dNTP}(2.5 \mathrm{mmol}$ /l); $5 \mu \mathrm{l}$ buffer solution; 1U Ex Taq DNA polymerase (Takara Bio Co.). The reaction conditions were: $95^{\circ} \mathrm{C} 5 \mathrm{~min}, 95^{\circ} \mathrm{C}$ $30 \mathrm{sec}, 55^{\circ} \mathrm{C} 30 \mathrm{sec}, 72^{\circ} \mathrm{C} 30 \mathrm{sec}, 32$ cycles; extension at $72^{\circ} \mathrm{C}$ for $5 \mathrm{~min}$. The PCR products were run on $1.5 \%$ agarose gel, visualized by goldview, and analyzed by UV analyzer.

PCR amplification. All exons of the DUOXA2 gene were amplified in patients first. If an exon mutation was identified in a patient, the target fragment was also amplified in the family members and in the 100 normal healthy controls.

Sequencing. PCR products were sent to Shanghai Sangon Biotechnology Co., Ltd., for sequencing using forward and reverse primers by AB13700 automatic sequence analyzer. The sequencing primers used were the same as the PCR primers. For samples with suspected mutations, amplification and sequencing were repeated three times. If the results were consistent, the mutation was considered real, otherwise it was considered as experimental error.
Statistical analysis. The statistical $\chi^{2}$ test was used and $\mathrm{P}<0.05$ was considered to indicate statistically significant differences.

\section{Results}

PCR products of 6 exons ran as bright single bands as visualized by UV analyzers after $1.5 \%$ agarose gel electrophoresis (Fig. 1). These 6 exon sequences were compared with GenBank DUOXA2 standard sequence (Gene ID, 405 753) using the NCBI BLAST program. The simultaneous presence of two mutations in the DUOXA2 gene in one $\mathrm{CH}$ child was identified in this study, a compound heterozygous mutation. The parents of the child were the carriers of the mutated gene (Fig. 2) and no corresponding mutations were found in the 100 normal controls, consistent with the recessive genetic law. The two mutations are an insertion of A base between 413 and $414 \mathrm{bp}$ on the 4 th exon (Fig. 3), and a $\mathrm{C} \rightarrow \mathrm{G}$ substitution on the 5th exon of 738 bp (p.Y246X). Aside from the $\mathrm{C} \rightarrow \mathrm{G}$ substitution on the 5 th exon of $738 \mathrm{bp}$, a $\mathrm{C} \rightarrow \mathrm{T}$ substitution was found in 4 patients and in 11 healthy controls, with a frequency of $4 / 47(8.5 \%)$ and $11 / 100(11 \%)$, respectively, which was not significantly different $(\mathrm{P}>0.05)$. This substitution did not cause amino acid change (Fig. 4). In addition, 3 patients and their family members and 9 normal controls had a $\mathrm{C} \rightarrow \mathrm{G}$ substitution on the 3rd exon of $278 \mathrm{bp}$ (Fig. 5), which is an SNP locus. The mutation frequency of this locus is $3 / 47(6.5 \%)$ in patients and 
A

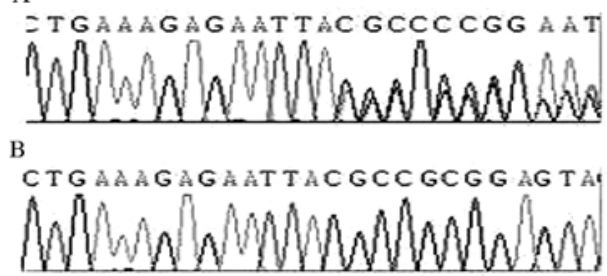

Figure 3. Two mutations are an insertion of A base between 413 and $414 \mathrm{bp}$ on the 4th exon. (A) A heterozygous insertion of A base on the 4th exon of 413-414 bp in the patient. (B) Normal control.

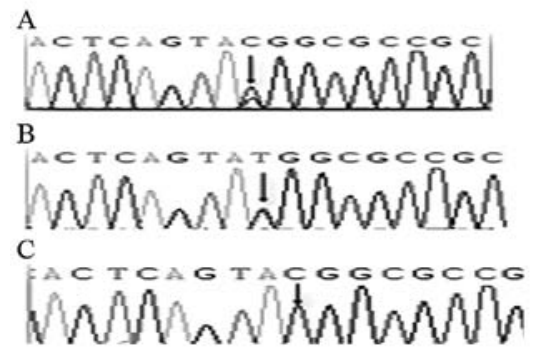

Figure 4. Heterozygous $\mathrm{C} \rightarrow \mathrm{G}$ mutation or $\mathrm{C} \rightarrow \mathrm{T}$ mutation. (A) A heterozygous $\mathrm{C} \rightarrow \mathrm{G}$ mutation on the 5th exon of 738 bp in the patient; arrow indicates the mutation site. (B) A heterozygous $\mathrm{C} \rightarrow \mathrm{T}$ mutation in 4 patients and in 11 healthy controls; arrow indicates the mutation site. (C) Normal control.

9/100 (9\%) in healthy controls, without significant difference between these two groups.

The $\mathrm{CH}$ patient, of Han ethnicity, with this novel DUOXA2 gene mutation was born in March 2010 at the Maternal and Child Health Hospital in Fujian Province. The parents were not diagnosed with any thyroid-related diseases. Following the initial screening of newborn diseases after birth, the child was brought to the hospital regularly for thyroid function tests and was diagnosed. This patient with the c.413-414insA (Y138X) mutation had mild $\mathrm{CH}$ symptoms.

\section{Discussion}

$\mathrm{CH}$ is one of the most common endocrine diseases in pediatrics. $\mathrm{CH}$ is caused by abnormal development due to various reasons such as athyreosis, hypoplastic or ectopic gland, and defects in thyroid hormone synthesis during embryonic development. This disease can lead to mental retardation and short stature, commonly known as cretinism. The average annual incidence rate of the disease is $1 / 1750-1 / 4000$, making it the most common congenital metabolic abnormality $(12,13)$.

In $\mathrm{CH}$ cases, approximately $15 \%$ are due to genetic defects of thyroid hormone synthesis, most of which are caused by iodine organification defects. The successful iodine organification requires the presence of $\mathrm{H}_{2} \mathrm{O}_{2}$, which is produced by NADPH oxidase. Duox 2 is essential for the catalytic activity of NADPH oxidase (3). Thus, abnormal Duox 2 function leads to the failure of iodine organification in the thyroid, affecting the synthesis of thyroid hormones, eventually resulting in reduced thyroid function.

Two genes were cloned and named DUOX maturation factors (DUOXA1 and DUOXA2) in Chicago in 2006. The DUOXA2 gene is located on chromosome 15 and consists

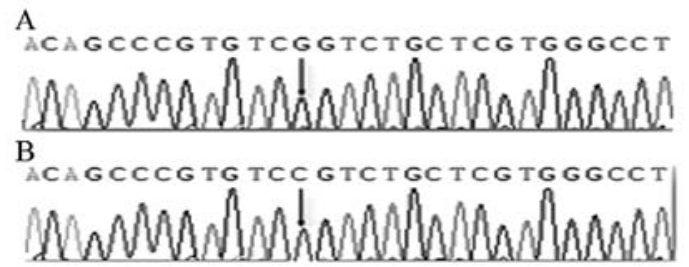

Figure 5. Homozygous $C \rightarrow G$ mutation. (A) Homozygous $C \rightarrow G$ mutation on the 3rd exon of 278 bp in 3 patients and their family members and in 9 healthy controls. (B) Normal control.

of 6 exons, encoding a 320 amino acid long transmembrane protein. DUOXA2 is mainly expressed in the thyroid and is weakly expressed in the epithelium of the digestive tract. DUOXA2 plays an important role in the process of Duox2 protein migration from the endoplasmic reticulum to the Golgi apparatus, in the maturation and transition to the apical membrane. As the DUOXA2 gene determines the normal functionality of Duox2, it is used as an important starting point for the molecular genetic study of $\mathrm{CH}$ (10).

In the present study, the 6 exons and their flanking regions of the DUOXA2 gene were subjected to sequence analysis. This is the first time that heterozygous mutations were found to occur simultaneously on the 4th and 5th exons. There was a heterozygous insertion of an A base between 413-414 bp on the 4th exon, generating a premature TAA stop codon and resulting in the early termination of protein translation. The $\mathrm{C} \rightarrow \mathrm{G}$ mutation on the 5 th exon at 738 bp leads to the generation of a premature stop codon (TAG). Screening of the 4th and 5th exons in the patient's parents and in the 100 healthy controls revealed that the patient's father is the carrier of the 413-414 bp insertion and the patient's mother is the carrier of the 738 bp $C \rightarrow G$ mutation; these two mutations were not found in the normal controls. This pattern is in line with the recessive inheritance law, thus we consider that this compound heterozygous mutation is the cause of the disease in this patient. The insertion mutation on the 4th exon of 413-414 bp was not found in the NCBI SNP database, therefore this is a novel finding. Our finding expands the mutation spectrum of the DUOXA2 gene.

The mutation on the 5th exon of 738 bp was an SNP locus (rs4774518) found in the NCBI database. It has been indicated that two mutations, the $\mathrm{C} \rightarrow \mathrm{G}$ and the $\mathrm{C} \rightarrow \mathrm{T}$ mutation, exist in this SNP. Among the 47 patients and the 100 normal controls in this study, we found a $\mathrm{C} \rightarrow \mathrm{G}$ heterozygous mutation in one patient only, and it was not found in the 100 healthy controls. The $\mathrm{C} \rightarrow \mathrm{T}$ mutation was found in patients and healthy controls, including 4 cases of patients $(4 / 47,8.5 \%$ ) and 11 cases of healthy controls $(11 / 100,11 \%)$. The difference between the two groups is not statistically significant $(\mathrm{P}>0.05)$, consistent with what was reported by Zamproni et al (11). In addition, a $\mathrm{C} \rightarrow \mathrm{G}$ homozygous mutation on the $3 \mathrm{rd}$ exon of $278 \mathrm{bp}$ in the DUOXA2 gene was found in 3 patients and in 9 healthy controls. It was verified as another SNP (rs2576090) in the NCBI database. Although the mutation led to a CGT $\rightarrow$ GGT change with a corresponding amino acid change, the present study suggests that the mutation frequency between patients and healthy controls was not statistically different, therefore we do not consider this mutation the cause of $\mathrm{CH}$. 
Of note, no mutations were found at the 1st and 2nd exons of the DUOXA2 gene. Only a point mutation was found in the flanking intron regions in 2 patients, indicating that segment of the sequence is relatively stable. Heterozygous or homozygous single nucleotide substitution polymorphisms were found at the 4 th and 5 th intron regions in 11 patients, at different sites. Whether the polymorphisms affect the RNA transcription process and lead to genetic instability of the DUOXA2 gene requires further study.

In conclusion, the present study reports a novel c.413-414insA (Y138X) mutation for $\mathrm{CH}$, which expands the mutational spectrum of the DUOXA2 gene. The potential function of this novel mutation requires further investigation.

\section{References}

1. Park SM, Clifton-Bligh RJ, Betts $\mathrm{P}$ and Chatterjee VK: Congenital hypothyroidism and apparent athyreosis with compound heterozygosity or compensated hypothyroidism with probable hemizygosity for inactivating mutations of the TSH receptor. Clin Endocrinol (Oxf) 60: 220-227, 2004.

2. Targovnik HM, Esperante SA and Rivolta CM: Genetics and phenomics of hypothyroidism and goiter due to thyroglobulin mutations. Mol Cell Endocrinol 322: 44-55, 2010.

3. Vaisman M, Rosenthal D and Carvalho D: Enzymes involved in thyroid iodide organification. Arq Bras Endocrinol Metabol 48: 9-15, 2004 (In Portuguese).

4. Vigone MC, Fugazzola L, Zamproni I, et al: Persistent mild hypothyroidism associated with novel sequence variants of the DUOX2 gene in two siblings. Hum Mutat 26: 395, 2005.
5. De Deken X, Wang D, Dumont J, et al: Characterization of ThOX proteins as components of the thyroid $\mathrm{H}(2) \mathrm{O}(2)$-generating system. Exp Cell Res 273: 187-196, 2002.

6. Moreno JC, Bikker H, Kempers MJ, et al: Inactivating mutations in the gene for thyroid oxidase 2 (THOX2) and congenital hypothyroidism. N Engl J Med 347: 95-102, 2002.

7. Varela V, Rivolta CM, Esperante SA, et al: Three mutations (p.Q36H, p.G418fsX482, and g.IVS19-2A $>$ C) in the dual oxidase 2 gene responsible for congenital goiter and iodide organification defect. Clin Chem 52: 182-191, 2006.

8. Hoste C, Rigutto S, Van Vliet G, et al: Compound heterozygosity for a novel hemizygous missense mutation and a partial deletion affecting the catalytic core of the $\mathrm{H}_{2} \mathrm{O}_{2}$-generating enzyme DUOX2 associated with transient congenital hypothyroidism. Hum Mutat 31: E1304-E1319, 2010.

9. Pfarr N, Korsch E, Kaspers S, et al: Congenital hypothyroidism caused by new mutations in the thyroid oxidase 2 (THOX2) gene. Clin Endocrinol (Oxf) 65: 810-815, 2006.

10. Grasberger $\mathrm{H}$ and Refetoff S: Identification of the maturation factor for dual oxidase. Evolution of an eukaryotic operon equivalent. J Biol Chem 281: 18269-18272, 2006.

11. Zamproni I, Grasberger H, Cortinovis F, et al: Biallelic inactivation of the dual oxidase maturation factor 2 (DUOXA2) gene as a novel cause of congenital hypothyroidism. J Clin Endocrinol Metab 93: 605-610, 2008.

12. Knobel M and Medeiros-Neto G: An outline of inherited disorders of the thyroid hormone generating system. Thyroid 13: 771-802, 2003

13. Zhu W, Wang Z, Chen $\mathrm{H}$, et al: Analysis of four-year congenital hypothyroidism neonatal screening in Jiudi City, Fujian Province. Zhong Guo You Sheng You Yu Za Zhi 12: 97-98, 2005. 